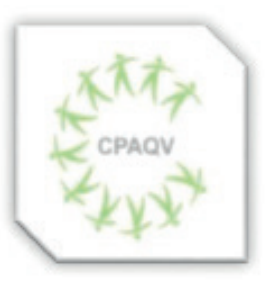

ISSN: 2178-7514

Vol. 13 | N. 2 | Ano 2021
ARTIGO ORIGINAL

\section{ANÁLISE DA SAÚDE MENTAL DOS PROFESSORES DE UMA INSTITUIÇÃO DE ENSINO SUPERIOR EM MEIO A PANDEMIA}

\section{Analysis of the mental health of teachers at a higher education institution in the midst of a pandemic}

Jade Gomes Da Costa Medeiros, Caroline de Souza Alovisi, Karla Gonçalves Godoy, Daiana Rossi Lima Marques Martins, Raquel Dutra Massad, Cíntia Regina De Carvalho França Araújo, Fabiana Costa Dos Santos, Lucas Rayan Gonçalves Ribeiro, Paula Beatriz Teixeira De Carvalho, Thamyris Raquel Alvez Cantarela, Saymon de Albuquerque, Arlindo Gonzaga Branco Junior, Luis Felipe Silio

\title{
RESUMO
}

Esse projeto se estabelece com o intuito de avaliar a qualidade da saúde psíquica de docentes de uma instituição do ensino superior no Norte do país, visto que já anterior à pandemia, a negligência ao cuidado com esses profissionais é presente, e atualmente diante do cenário instaurado da pandemia da COVID-19 esse quadro tem sido acentuado para muitos profissionais. Nesse contexto, percebeu-se no estudo que mais que três quartos dos entrevistados sentem que sua jornada de trabalho foi ampliada. Vale destacar ainda, que referente a busca por apoio psicológico em unanimidade, nenhum dos docentes vivenciou tal experiencia. Tendo em vista as diversas motivações para tal feito variaram desde relatarem falta de tempo a não sentirem necessidade do suporte. Por fim, através do estudo foi possível depreender que o cenário pandêmico associado as mudanças impostas, contribuíram para desafiar ainda mais o ambiente acadêmico associado aos docentes e suas nuances.

Palavras-chave: Saúde Mental. Docentes. Coronavírus

\begin{abstract}
This project is established with the aim of evaluating the quality of the psychic health of professors at a higher education institution in the North of the country, since before the pandemic, negligence in the care of these professionals is present, and currently faced with the established scenario of the COVID-19 pandemic, this picture has been accentuated for many professionals. In this context, it was noticed in the study that more than three quarters of respondents feel that their working hours have been extended. It is also worth noting that regarding the unanimous search for psychological support, none of the professors experienced such an experience. In view of the various motivations for such a feat, they ranged from reporting lack of time to not feeling the need for support. Finally, through the study it was possible to infer that the pandemic scenario associated with the imposed changes, contributed to further challenge the academic environment associated with teachers and its nuances.
\end{abstract}

Keywords: Mental Health. Teachers. Coronavirus

Autor de correspondência

Luis Felipe Silio - E-mail: silioeducafisica@gmail.com 


\section{INTRODUÇÃO}

Em 31 de dezembro de 2019 foi identificada pela primeira vez na cidade de Wuhan, na China, a doença causada pelo Coronavírus (COVID-19), e em janeiro de 2020 a Organização Mundial de Saúde declarou esta patologia como uma emergência em saúde pública a nível internacional. ${ }^{1}$

Desde a sua descoberta, a pandemia causada pela COVID-19 tem se alastrado de maneira devastadora, gerando impactos diversos na sociedade mundial, relacionados à morbimortalidade e adoção de medidas de isolamento de toda população a fim de conter a disseminação do vírus. ${ }^{2}$

De acordo com os dados do Ministério da Saúde, o Brasil ocupa a segunda posição no mundo em relação ao número de casos e ao registro de óbitos.

Tendo em vista o novo contexto mundial provocado pela SARS-CoV-2, foi preciso que os gestores das faculdades e universidades colocassem em prática as normativas preconizadas pela Portaria no 345/2020 do pelo Ministério da Educação ${ }^{3}$ que autoriza, em caráter excepcional, a substituição das disciplinas presenciais, em andamento, por aulas que utilizem meios e tecnologias de informação e comunicação, a fim de se manter a continuidade ao semestre e, consequentemente, ano letivo. ${ }^{4}$

Diante de pressões tanto das instituições para o alcance dos objetivos, quanto das exigências dos alunos para um melhor aproveitamento, os docentes acabam desenvolvendo quadros de estresse e ansiedade que podem culminar no esgotamento profissional ou em depressão. Dentre as dificuldades de um ensino a distância (EAD) está a falta de domínio tecnológico, problemas na conexão com a internet, confecção de material didático digital, postagem de arquivos e aulas, gravações de aulas de conteúdos com carga horária densa ou pouco maleável para modalidade EAD, tempo extra para a organização e o planejamento das atividades, além de ter que conciliar essa nova modalidade a sua rotina home office.

Baseado no supracitado este trabalho tem por objetivo avaliar a qualidade de saúde psíquica de docentes de uma instituição de ensino superior em meio a pandemia do Covid-19.

\section{MÉTODOS}

Trata-se de um trabalho exploratório, descritivo e quantitativo que ocorreu em uma universidade privada de Porto Velho, Rondônia.

O projeto foi aplicado por meio de um questionário adaptado para nosso cenário composto por 15 perguntas acerca de sua vivência na modalidade de ensino REAR (Regime Especial de Aprendizagem Remota) proposto pela instituição em período de pandemia pelo COVID-19 baseado no estudo de Brief Symptom Inventory (BSI 18) ${ }^{5}$ e todos os possíveis comprometimentos psíquicos, além de perguntas de cunho socioeconômicas, sociodemográficas. 
A aplicação do questionário ocorreu de forma virtual, por meio de convite online acesso ao Google Forms, de modo a garantir o sigilo, evitando possíveis interferências e constrangimentos, tendo em vista as limitações de saúde pública impostas pela pandemia do SARSCoV2 pelo não retorno às atividades presenciais da instituição, obedecendo os protocolos de biossegurança atualizado pela ANVISA (Agência Nacional de Vigilância Sanitária). ${ }^{6}$

Feito a coleta, as informações obtidas foram compiladas em tabelas no editor de planilhas Microsoft Office Excel, e então analisadas e comparadas a feito de observar a prevalência das principais queixas.

O projeto foi aprovado pelo comitê de ética em pesquisa do centro universitário e São Lucas sob parecer número 4.581.683.

\section{RESULTADOS}

Participaram da coleta 25 docentes ao total, sendo a média das idades correspondeu a 40 anos, com a menor faixa etária representada por 25 anos e a maior por 65 anos. Analisando o estado civil dos entrevistados 68,0 \% casados, $24,0 \%$ são solteiros e 8,0 \% divorciados e não houve registro de viúvos.

Avaliando a orientação sexual dos entrevistados, 92\% declararam ser heterossexual e $8 \%$ se declararam homossexual. Analisando a renda familiar desses docentes, 88\% possui de 5 salários mínimos ou mais e $12 \%$ até 4 salários mínimos. Estes dados combinados nos afirmam que a amostra aqui analisada faz é composta por indivíduos de classe média.

Com relação a raça, $56 \%$ se consideram pardos, 36\% se consideram brancos, $4 \%$ se consideram amarelos e 4\% se consideram pretos. Em relação a frequência com que os docentes têm vivenciado o sentimento de esgotamento físico e/ ou mental devido ao trabalho tem-se que 32\% consideram frequentemente, $28 \%$ consideram algumas vezes, $24 \%$ consideram sempre e $16 \%$ consideram raramente.

Quando indagados sobre as cobranças acadêmicas impostas pela instituição no período de pandemia, 14 participantes avaliaram como normais, 9 como excessivas, 1 participante avaliou como excessiva e sem nexo e outro participante avaliou as cobranças como incoerentes. Quando questionados em relação às horas dedicadas ao trabalho, comparando com o período anterior a pandemia e o atual, $76 \%$ dos participantes declararam tempo de trabalho ampliado, 12\% declararam que o tempo é semelhante e 12\% disseram que o tempo de trabalho é reduzido.

Ademais, quando questionados sobre a experiência de trabalhar no ensino a distância e sobre sua saúde emocional no exato momento em comparação em um período pré-pandemia, foi utilizado uma escala de 0 a 10 , sendo 0 péssimo e 10 excelente, e transformados em porcentagens. Para a experiência de trabalhar no ensino a distância encontramos apenas 10\% que deram nota máxima e 1\% para pior nota, no 
entanto somando temos $84 \%$ das pessoas dando notas positivas. Sobre a saúde emocional surgiu uma igualdade, representando $20 \%$ para nota 4 (escala negativa) e para nota 8 (escala positiva).

Ao serem interrogados acerca da motivação como profissional para lecionar nesse período de pandemia, em relação à fase anterior ao isolamento social, $40 \%$ relataram piora na motivação profissional e cansaço durante o período de pandemia, 28\% mencionaram que a motivação atual é semelhante ao período anterior a pandemia, 16\% afirmam que conseguiram adaptar a motivação apesar das desvantagens e desafios, e outros $16 \%$ se sentem mais motivados durante esse período de pandemia, citam que a mudança os motivou a aprender muito mais como profissionais.

No que tange ao otimismo dos professores sobre a volta às aulas presenciais, ocorreu um empate em relação aos que se demonstraram otimistas e os que não estão otimistas, cada grupo com $44 \%$ e $12 \%$ dos docentes foram indiferentes. Em relação ao pensamento sobre desistir de lecionar devido às dificuldades enfrentadas durante esse atual cenário de pandemia, 48\% dos docentes relataram que nunca pensaram em desistir, 32\% algumas vezes, 16\% raramente e $4 \%$ frequentemente. Nenhum docente assinalou a opção sempre.

Sobre a realização profissional, aproximadamente $\quad 65 \%$ dos professores entrevistados declaram serem realizados, dividindo alguns com realização plena, e outros realizados mesmo diante de tantas dificuldades que estão enfrentando desde o início da pandemia. Dos 35\% restantes, que negam se sentirem realizados com a sua profissão diante deste cenário, alegam estagnação, desvalorização e insatisfação financeira.

Ao serem questionados se procuraram ajuda psicológica na Instituição em algum momento durante a pandemia, 100\% dos docentes relataram que não. Porém, 20\% (5) alegaram que fazem acompanhamento psicológico externamente. Os 80\% restantes declararam a não procura por ajuda psicológica por diversos motivos como: não sentir necessidade até o momento $(8=32 \%)$, falta de tempo pela correria do dia a dia $(3=12 \%)$, respeito ao distanciamento $(1=4 \%)$, pela dificuldade causada pela pandemia $(1=4 \%)$, por não saber do serviço disponibilizado $(1=4 \%)$ entre outros não justificados $(6=24 \%)$.

Quando perguntados se a rede de ensino estava oferecendo amparo para atuar a distância, $80 \%$ dos docentes entrevistados responderam que sim e citaram o uso de plataformas digitais disponibilizadas pela instituição, incluindo auxílio de forma geral. Além disso, 8\% dos docentes responderam que não. Entretanto, 4\% desse valor justificou que não recebia esse amparo por ser profissional que atua em aula prática. Ademais, $12 \%$ abstiveram-se de responder à pergunta. 


\section{DISCUSSÃO}

A partir dos dados obtidos, a média da idade dos docentes apresentou-se na faixa dos 40 anos, sendo, portanto, sua grande maioria representados por um grupo que em apesar de acompanhar a implementação da internet no país, por vezes pode vivenciar algum grau de resistência às mudanças pedagógicas tecnodigitais abruptas impostas pela pandemia, a exemplo do que foi descrito por Santos et $\mathrm{al}^{7}$ que pode acarretar em ainda mais dificuldades para adequação a essa fase vigente. Ademais, ao analisar o estado civil dos entrevistados, em que o perfil predominante são dos casados, é possível depreender como explicitado por Pereira et al8, que a desconfiguração vivenciada pelo ambiente de trabalho migrando para o meio domiciliar e as novas dinâmicas que foram inseridas influenciaram não apenas o docente mas seu núcleo familiar e possivelmente transferiram a tensão e estresse profissional para o ambiente do lar e possivelmente para maiores desafios familiares, como abordado por Lebow ${ }^{9}$ que as consequências enfrentadas muitas vezes pelo trabalho tem trazido um aumento de atritos e até mesmo divórcios no período de pandemia.

Tendo em vista que a maior parte do grupo entrevistado pertence ao sexo feminino, pode-se inferir que de acordo com isso algumas características identificadas por esse sexo pode tornar a mulher nesse momento de pandemia um grupo mais acometido pelo processo do confinamento $^{10}$, pois além do serviços profissionais muitas delas podem exercer diferentes funções sociais como mãe, dona de casa, e assim tendo inúmeras obrigações atrelado ainda a Jornada de trabalho que por serem todas no mesmo ambiente, corrobora para um maior estresse das entrevistadas.

O cenário provocado pela pandemia fez com que os gestores das faculdades e universidades tivessem que colocar em prática as normativas do Ministério da Educação que autorizou a substituição das disciplinas presenciais para um modelo de educação remota enquanto durasse a crise sanitária. Os desafios de um ensino à distância emergencial levaram mais da metade $(52 \%)$ dos docentes de medicina da Universidade em questão a pensar em desistir de lecionar em algum momento durante esse atual cenário de pandemia. A situação de confinamento provoca um conjunto alargado de estados emocionais negativos, que foi evidenciado em um estudo chinês $^{11}$ que revelou inúmeros docentes adoecidos mentalmente pela Covid-19, devido a transtorno depressivo leve, transtorno afetivo bipolar, ansiedade generalizada, transtorno de adaptação e síndrome de Burnout ou síndrome do esgotamento profissional. ${ }^{12}$ Verificou- se que inúmeros docentes acabaram adoecendo por não conseguirem atingir os objetivos propostos pela instituição, devido às diversas pressões relacionadas ao manuseio das tecnologias e gravações de aulas e a estrutura inadequada das instituições de ensino e evasão do aluno. 
O estudo de Santos $^{13}$ relatou várias dificuldades enfrentadas pelos professores devido a virtualização do processo educacional como a ausência de interação e de relacionamento interpessoal natural e físico entre professor e aluno, a maior carga horária de trabalho e a incompatibilidade do home office com a vida pessoal pois os docentes tiveram que transferir e adaptar o trabalho no domicílio. Em relação aos aspectos tecnológicos, o estudo destaca a falta de recursos e a tecnofobia e em relação ao aspecto pedagógico foi verificado uma fragilidade no desenvolvimento de competências direcionadas às estratégias metodológicas e práticas de ensino da educação on-line.

A partir da coleta e análise dos dados acerca da motivação para lecionar no período de pandemia, notou-se que aproximadamente $40 \%$ dos docentes sentem-se desmotivados profissionalmente, por inúmeros motivos e desafios impostos, fato este descrito também por Mckimm et al. $(2020)^{14}$, que afirmam que essa adesão urgente e apressada ao ensino a distância para atender a demanda caótica do momento, impôs adversidades atípicas, marcando os professores com medo, incertezas, dúvidas e diversas expectativas, pois foram inseridos em um meio totalmente diferente e sem treinamento prévio e precisam apesar de tudo isso, se reinventar e inovar suas estratégias pedagógicas diariamente. ${ }^{15}$ Esse cenário atual de crise corrobora ainda mais com o adoecimento físico e mental dos docentes, visto que além das pressões pessoais, existe a pressão para atingir os objetivos impostos pelos gestores educacionais, a culpa pela estrutura inadequada oferecida pelas instituições para lecionar as aulas de forma remota e ainda precisam lidar com a evasão dos discentes que não estão satisfeitos com o método de ensino. Ademais, tudo isso em conjunto culmina na desmotivação do profissional da educação frente aos desafios impostos em tempos de pandemia Covid-19.

Sob o aspecto da experiência do ensino a distância que os profissionais docentes estão sendo sujeitos, o resultado mostra 84\% para valores positivos, configurando que apesar das dificuldades em meio a pandemia, estes profissionais afirmam uma boa experiência, o que apresenta uma diferença em estudo8 realizado em Portugal mostrou que as aulas à distância são experiências difíceis e negativas, revelando o cansaço e desmotivação dos professores para lecionar em ambiente remoto. E a percepção dessa condição entre professores no Brasil foi verificada por meio de uma nuvem de palavras, sendo traduzidas por termos como: ansiedade, pânico, cansaço, desafio, exaustão, incerteza, insegurança, medo e sobrecarga de trabalho.

A saúde mental sempre será uma abordagem prevista em docentes, pois estes estão frequentemente sendo sujeitos ao desequilíbrio emocional, proveniente de fatores como longas jornadas de trabalho e baixa valorização no mercado. Isso implica a uma falta de confiança e que leva a sentimentos de preocupação, medo, 
ansiedade e exaustão, sendo ainda que muitos negam o assunto ou consideram insignificantes. Em nossos resultados encontramos uma porcentagem igualitária no aspecto positivo e negativo de sua saúde mental pré-pandemia. Em uma situação anterior a pandemia ${ }^{16}$ descreve-se que a extensa e prolongada jornada de trabalho provoca além de uma série de sofrimento psíquicos e físicos, o desmantelamento de uma profissão que sempre ocupou um lugar de reconhecimento. Muitos procuram outras atividades com o intuito de preservar a saúde mental. E que outros sentem que o adoecimento mental especialmente provocado pelo auto aceleração e controle da gestão, desgasta sua vida pessoal e profissional. Da mesma forma, em tempo de pandemia, encontramos estudos nacionais e internacionais demonstrando o surgimento de vários sintomas psicopatológicos em docentes de ensino à distância.

Depreende-se que devido à complexidade de sua multitarefa, o ensino está entre as atividades de trabalho mais estressantes. A probabilidade de professores desenvolverem estresse, depressão e ansiedade é duas vezes maior quando comparada a outras profissões e que no Brasil, esses profissionais ocupam o segundo lugar na categoria de doenças ocupacionais. Cita também que na China, vários professores adoecem mentalmente devido à pandemia de Covid-19, apresentando transtorno depressivo leve, transtorno afetivo bipolar, ansiedade generalizada, transtorno de adaptação e Síndrome de Burnout. ${ }^{8}$
Diante da coleta de dados 34\% dos docentes avaliaram as cobranças impostas pelas instituições como excessivas e 76\% afirmaram que a carga de trabalho nesse período pandêmico foi ampliada, situação semelhante descrita por Souza et al. ${ }^{17}$, a qual relatou que a produtividade do trabalho de docentes de escolas privadas era medida pela quantidade de atividades concluídas pelos alunos nas plataformas e avaliações de aprendizagem aplicadas. ${ }^{18}$ Diante desse cenário, essa conjuntura de exigências de desempenho em demasia, a falta de tempo de descanso por cargas horárias estendidas de trabalho dentre outras adversidades emocionais, sociais e interpessoais contribuem negativamente para a saúde mental dos professores. Além disso, esses docentes podem ter sua vida profissional comprometida, pois a intensificação do trabalho do docente mediante a falta de tempo para esse fim, afeta a atualização e requalificação desses profissionais para o mercado de trabalho ${ }^{19}$.

Em relação ao otimismo sobre o retorno às aulas presenciais houve um empate em relação aos que se demonstraram otimistas e os que não estão otimistas (44\%), o que pode ser um reflexo da segunda onda de Covid-19 no Brasil em meio ao caos instalado pela primeira onda da doença. A necessidade do distanciamento social como meio de combate ao aumento do número de casos de pacientes infectados com Coronavírus, além da preocupação com o surgimento das novas variantes do vírus, faz com que medidas rígidas de controle da doença sejam adotadas pelos governantes. 
O ensino remoto emergencial estabelecido pela pandemia COVID-19 tem exigido do docente, além dos seus métodos pedagógicos, aprender sozinho sobre as Tecnologias da informação e comunicação (TICs) ${ }^{20}$ e realizar gravações das aulas em seu domicílio. Somado a isso, o docente tem desempenhado importante papel motivador, guia acadêmico e conselheiro espiritual dos estudantes, ajudando-os na contenção afetiva e na prática da resiliência ${ }^{21,22}$. Como resultado à complexidade de suas diversas funções, à docência se enquadra entre as atividades laborais mais estressantes ${ }^{23}$.

Alguns trabalhos apontaram que a probabilidade de professores desenvolverem estresse, e doenças como depressão e ansiedade é duas vezes maior quando comparados às demais profissões há Relatos que no Brasil, os docentes ocupam o segundo lugar na categoria das doenças ocupacionais. Alguns transtornos e doenças associadas à conjuntura trabalhista expõem determinações diretas proveniente das novas estruturas e constituições do mundo do trabalho $22,23,24,25$. Nesse contexto, a literatura que avalia a relação entre o meio de trabalho e os impactos na saúde mental demonstram que a situação de exploração e fragilidades das condições trabalhistas acarretam prejuízos à saúde de docentes e trabalhadores da educação24, evidenciando o adoecimento expresso pela Síndrome de Burnout, caracterizada pelo esgotamento profissional ${ }^{26}$.
Sanchez et al. ${ }^{15}$ observaram em seu estudo que a carga horária média de trabalho semanal do professor era de 32,5 horas. Porém, na atual condição de pandemia, 48,3\% dos entrevistados confirmaram carga horária de 40 horas semanais, havendo relatos de carga horária superior a 45 horas por semana.

Em relação à realização profissional, 35\% dos entrevistados negam sentirem-se realizados com a docência, por motivos diferentes, mas que se ligam entre si, como a desvalorização e estagnação. Assim, fica claro que as dificuldades expostas pela pandemia aumentaram exponencialmente essa insatisfação, já que, ser professor no Brasil sempre foi um grande desafio, mas diante das barreiras para o desenvolvimento desta nova forma de ensino, muitos profissionais apesar de amarem o que fazem sentem-se frustrados. ${ }^{27}$ Quando lhe é apresentada uma proposta de mudança, certamente o professor sofre uma desestabilização em suas crenças e práticas, o novo provoca-lhe conflito. A mudança se introduz em um espaço de contradição em que o professor avalia sua utilidade e o grau de esforço que lhe é exigido.

Nesse cenário, $80 \%$ dos docentes entrevistados responderam que estavam sendo amparados pela instituição de ensino para atuar a distância. Além disso, essa parcela utilizou como exemplo o uso de plataformas digitais e o auxílio disponibilizado de forma geral. Entretanto, é essencial destacar a necessidade de um olhar mais abrangente para a saúde mental desses 
docentes, tendo em vista que $12 \%$ abstiveram-se de responder e $8 \%$ demonstraram não receber o amparo necessário. Com isso, é fundamental a criação de uma rede de ensino com amparo, através de atendimentos periódicos por psicólogos capacitados e a abertura de diálogo direto (por meio de reuniões frequentes através da criação de espaços virtuais) com os gestores responsáveis, tendo como objetivo uma maior união do corpo pedagógico e a minimização de angústias provocadas pela pandemia. Assim, poder-se-á amenizar a problemática.

No que tange sobre o esgotamento mental sofrido pelos docentes dessa instituição, $32 \%$ têm esse sentimento de forma frequente, $24 \%$ dizem que sempre e $28 \%$ apontam algumas vezes e, somente, $16 \%$ afirmam o sentimento de esgotamento mental ser raro. Desse modo, no geral, é visto que essa questão está sendo constantemente presente na vida desses professores.

Dentro disso, há outra dificuldade a qual é exigir que esses professores aprendam a lidar com novas tecnologias e formas de ensinar em plataformas digitais, visto que, atualmente a sala de aula foi substituída por uma sala virtual, dificultando, ainda mais a interação entre mestre e pupilo. Outrossim, a insegurança vivenciada diariamente pelo medo do vírus ter a possibilidade de contaminar a si e sua família trás o adoecimento mental ${ }^{23}$.

Existem estudos internacionais que apontam a síndrome do esgotamento profissional já como uma realidade no mundo, juntamente, ao estresse, ansiedade e outros distúrbios como a depressão ${ }^{26}$. Por outro lado, o esgotamento mental também existe devido à "escravização digital” que está sendo colocada, pois, as jornadas de trabalho se tornaram aumentadas em relação ao que tinham quando exerciam a metodologia presencial. ${ }^{17}$

\section{CONCLUSÃO}

Vê-se, portanto, que nesse estudo foi discutido acerca da saúde mental dos docentes de ensino particular superior amazônico, frente a vigência da pandemia instaurada e os possíveis impactos que tal cenário pôde provocar aos envolvidos. Desse modo, discorreu-se acerca dos fatores socioeconômicos, mas ademais sobre questões intrínsecas as impressões do universo educacional e também ao modo como estes, temse observado durante a pandemia como indivíduos e profissionais. Em razão disso, tal pesquisa é um meio de registro de como ser educador no Brasil e principalmente na quarentena tem sido um quadro desafiador, e por vezes, negligenciado. Em consonância, a análise e observação dos dados obtidos servem como contribuição para fomentar indagações ou mesmo intervenções nos ambientes científicos e acadêmicos em que diversos profissionais se encontram. No intuito, de haver uma redução da incidência e prevalência de desequilíbrios emocionais derivados do ambiente profissional e principalmente garantir a qualidade de vida aos professores. 


\section{REFERENCIAS}

1. World Health Organization. (2020a). Mental health and psychosocial considerations during the COVID-19 outbreak. Geneva: Author. Disponível em: https://www. who.int/docs/default-source/coronaviruse/mentalhealthconsiderations.pdf.

2. Silva AFD, Estrela FM, Lima NS, Abreu CTDA. Saúde mental de docentes universitários em tempos de pandemia. Physis. 2020;30(2):e300216.

3. PORTARIA MEC No 345 , DE 19 DE MARÇO DE 2020 - https://abmes.org.br/arquivos/legislacoes/ Portaria-mec-345-2020-03-19.pdf

4. Jowsey T, Foster G, Cooper-Ioelu P, Jacobs S. Blended learning via distance in pre-registration nursing education: A scoping review. Nurse Education in Practice. março de 2020; 44:102775.

5. Nazaré B, Pereira M, Canavarro MC. Avaliação breve da psicossintomatologia: Análise fatorial confirmatória da versão portuguesa do Brief Symptom Inventory 18 (BSI 18). Análise Psicológica. 5 de junho de 2017;35(2):213-30. 6. ANVISA - Agência Nacional de Vigilância Sanitária. Nota Técnica $n^{\circ}$ 04/2020 GVIMS/GGTES/ANVISA. Orientações para Serviços de Saúde:medidas de prevenção e controle que devem ser adotadas durante a assistência aos casos suspeitos ou confirmados de infecção pelo novo coronavírus. 2020.

ab598660-3de4-4f14-8e6f-b9341c196b28

7. Santos GMRF dos, Silva ME da, Belmonte B do R. COVID-19: emergency remote teaching and university professors' mental health. Rev Bras Saude Mater Infant. fevereiro de 2021;21(suppl 1):237-43.

8. PEREIRA, Hortência Pessoa, SANTOS, Fábio Viana, MANENTI, Mariana Aguiar. SAÚDE MENTAL DE DOCENTES EM TEMPOS DE PANDEMIA: OS IMPACTOS DAS ATIVIDADES REMOTAS. $1^{\circ}$ de setembro de 2020 [citado 23 de junho de 2021]; Disponível em: https://zenodo.org/record/3986851

9. Lebow JL. The Challenges of COVID $\square 19$ for Divorcing and Post $\square$ divorce Families. Fam Proc. Setembro de 2020;59(3):967-73.

10. Lemos AHDC, Barbosa ADO, Monzato PP. MULHERES EM HOME OFFICE DURANTE A PANDEMIA DA COVID-19 E AS CONFIGURAÇÕES DO CONFLITO TRABALHO-FAMÍLIA. Rev adm empres. dezembro de 2020;60(6):388-99.

11. Wang J, Wang Z. Strengths, Weaknesses, Opportunities and Threats (SWOT) Analysis of China's Prevention and Control Strategy for the COVID-19 Epidemic. Int J Environ Res Public Health. 26 de março de 2020;17(7):E2235.

12. Silva SMF, Oliveira Á de F. Burnout em professores universitários do ensino particular. Psicol Esc Educ. 2019;23:e187785.

13. Santos HMR dos. Os desafios de educar através da Zoom em contexto de pandemia: investigando as experiências e perspectivas dos docentes portugueses. Prax Educ. 2020; 15:1-17.

14. McKimm J, Gibbs T, Bishop J, Jones P. Health Professions' Educators' Adaptation to Rapidly Changing Circumstances: The Ottawa 2020 Conference Experience. MedEdPublish [Internet]. 2020 [citado 23 de junho de 2021];9(1). Disponível em: https://www.mededpublish. org/manuscripts/2936.

15. Sanchez HM, Sanchez EG de M, Barbosa MA,
Guimarães EC, Porto CC. Impacto da saúde na qualidade de vida e trabalho de docentes universitários de diferentes áreas de conhecimento. Ciênc saúde coletiva. novembro de 2019;24(11):4111-23.

16. Leila Sueli Dillmann. O adoecimento no trabalho do ensino à distância: revelação de professorestutores [Internet] [Monografia (Especialização em Psicologia)]. Universidade Federal do Rio Grande do Sul; Disponível em: https://www.lume.ufrgs.br/bitstream/ handle $/ 10183 / 70052 / 000875613$.pdf?sequence $=1$

17. Souza KR de, Santos GB dos, Rodrigues AM dos S, Felix EG, Gomes L, Rocha GL da, et al. Trabalho remoto, saúde docente e greve virtual em cenário de pandemia. Trab educ saúde. janeiro de 2021;19:e00309141.

18. NEVES, M.Y.R.; SILVA, E.S. A dor e a delícia de ser (estar) professora: trabalho docente e saúde mental. Estudos e Pesquisas em Psicologia, Rio de Janeiro, v. 6, n. 1, p. 63-75, 2006.

19. HYPOLITO, Álvaro M.; VIEIRA, Jarbas S.; PIZZI, Laura C. V. Reestruturação curricular e autointensificação do trabalho docente. Currículo sem Fronteiras, v. 9, n. 2, p. 100-112, jul./dez. 2009. Disponível em: http://www. curriculosemfronteiras.org/vol9iss2articles/hypolitovieira-pizzi.pdf

20. Bezerra NPX, Veloso AP, Ribeiro E. Ressignificando a prática docente: experiências em tempos de pandemia. Rev Pemo. 3 de janeiro de 2021;3(2):323917.

21. Expósito CD, Marsollier RG. Virtualidad y educación en tiempos de COVID-19. Un estudio empírico en Argentina. educ.humanismo [Internet]. 24 de agosto de 2020 [citado 23 de junho de 2021];22(39). Disponível em: http://revistas.unisimon.edu.co/index.php/educacion/ article/view/4214

22. VILLAFUERTE J, Bello J, Cevallos Y, Bermello J. Rol de los docentes ante la crisis del COVID-19, una mirada desde el enfoque humano. REFCalE. 2020; 8 (1): 13450. Disponível em:https://www.scielo.br/pdf/rbsmi/ v21s1/1519-3829-rbsmi-21-s1-0237.pdf.

23. Silva AFD, Estrela FM, Lima NS, Abreu CTDA. Saúde mental de docentes universitários em tempos de pandemia. Physis. 2020;30(2):e300216.

24. Nunes Baptista M, Pereira Soares TF, Raad AJ, Milani Santos L. Burnout, estresse, depressão e suporte laboral em professores universitários. Rev Psicol, Organ Trab. 2019;19(1):564-70.

25. Moreira DZ, Rodrigues MB. Saúde mental e trabalho docente. Estud psicol (Natal). setembro de 2018;23(3):23647.

26.Araújo FJ de O, de Lima LSA, Cidade PIM, Nobre CB, Neto MLR. Impact Of Sars-Cov-2 And Its Reverberation In Global Higher Education And Mental Health. Psychiatry Research. junho de 2020;288:112977.

27. Márcia Silva do Carmo Brito V, Oliveira Brandão Veiga E, Magnelli Mangiavacchi B, Santos Curcio F. ENSINO REMO'TO, PRÁTICA DOCENTE E SAÚDE MENTAL EM TEMPOS DE PANDEMIA DA COVID-19: NOTAS INTRODUTÓRIAS. In 2020 [citado 23 de junho de 2021]. Disponível em: https://www.even3.com.br/anais/ coninter2020/296593

OBSERVAÇÃO: Os autores declaram não existir conflitos de interesse de qualquer natureza. 